\title{
Existence of Nontrivial Solution for a Nonlocal Elliptic Equation with Nonlinear Boundary Condition
}

\section{Fanglei Wang and Yukun An}

Department of Mathematics, Nanjing University of Aeronautics and Astronautics, Nanjing 210016, China

Correspondence should be addressed to Fanglei Wang, wang-fanglei@hotmail.com

Received 15 December 2008; Accepted 17 February 2009

Recommended by Zhitao Zhang

In this paper, we establish two different existence results of solutions for a nonlocal elliptic equations with nonlinear boundary condition. The first one is based on Galerkin method, and gives a priori estimate. The second one is based on Mountain Pass Lemma.

Copyright (C) 2009 F. Wang and Y. An. This is an open access article distributed under the Creative Commons Attribution License, which permits unrestricted use, distribution, and reproduction in any medium, provided the original work is properly cited.

\section{Introduction}

In this paper, we deal with the following elliptic equation with nonlinear boundary condition:

$$
\begin{gathered}
-\Delta u+u=\frac{f(x, u)}{M\left(\int_{\Omega}|\nabla u|^{2}+|u|^{2} d x\right)}, \quad \text { in } \Omega, \\
\frac{\partial u}{\partial \gamma}=g(x, u), \quad \text { on } \partial \Omega,
\end{gathered}
$$

where $\Omega$ is a bounded domain in $R^{N}$ with smooth boundary $\partial \Omega, N>2, \partial / \partial \gamma$ is the outer unite normal derivative, $M: R^{+} \rightarrow R$ is continuous, $f: \Omega \times R \rightarrow R, g: \partial \Omega \times R \rightarrow R$ are Carathéodory functions.

For (1.1), if the nonlocal term $M\left(\int_{\Omega}|\nabla u|^{2}+|u|^{2} d x\right)$ is replaced by $M\left(\int_{\Omega}|\nabla u|^{2} d x\right)$, then the equation

$$
-M\left(\int_{\Omega}|\nabla u|^{2} d x\right) \Delta u=f(x, u), \quad \text { in } \Omega
$$


is related to the stationary analog of the Kirchhoff equation:

$$
u_{t t}-M\left(\int_{\Omega}\left|\nabla_{x} u\right|^{2} d x\right) \Delta_{x} u=f(x, t),
$$

where $M(s)=a s+b, a, b>0$. It was proposed by Kirchhoff [1] as an extension of the classical D'Alembert wave equations for free vibrations of elastic strings. The Kirchhoff model takes into account the length changes of the string produced by transverse vibrations. Equation (1.3) received much attention and an abstract framework to the problem was proposed after the work [2]. Some interesting and further results can be found in $[3,4]$ and the references therein. In addition, (1.2) has important physical and biological background. There are many authors who pay more attention to this equation. In particularly, authors concerned with the existence of solutions for (1.2) with zero Dirichlet boundary condition via Galerkin method, and built the variational frame in [5,6]. More recently, Perera and Zhang obtained solutions of a class of nonlocal quasilinear elliptic boundary value problems using the variational methods, invariant sets of descent flow, Yang index, and critical groups $[7,8]$.

If the nonlocal term $M\left(\int_{\Omega}|\nabla u|^{2}+|u|^{2} d x\right)$ is replaced by $M\left(\int_{\Omega}|u|^{2} d x\right)$, then the equation

$$
-M\left(\int_{\Omega}|u|^{2} d x\right) \Delta u=f(x, u), \quad \text { in } \Omega
$$

arises in numerous physical models such as systems of particles in thermodynamical equilibrium via gravitational (Coulomb) potential, 2-D fully turbulent behavior of real flow, thermal runaway in Ohmic Heating, shear bands in metal deformed under high strain rates, among others. Because of its importance, in $[9,10]$, the authors similarly studied the existence of solution for (1.4) with zero Dirichlet boundary condition.

On the other hand, elliptic equations with nonlinear boundary conditions have become rather an active area of research; see [11-15] and reference therein. Those references present necessary and sufficient conditions of solutions of elliptic equations with nonlinear boundary conditions. In [13], the authors study the elliptic equation

$$
\Delta u=f(x, u), \quad \text { in } \Omega,
$$

with the nonlinear boundary condition

$$
\frac{\partial u}{\partial \gamma}=g(x, u), \quad \text { on } \partial \Omega
$$

They obtain various existence results applying coincidence degree theory and the method of upper and lower solutions.

Inspired by the above references, we deal with the existence of solutions for elliptic equation (1.1) with nonlinear boundary condition based on Galerkin method and the Mountain Pass Lemma.

The paper is organized as follows. In Section 2, we will give the existence of solution for (1.1) via Galerkin method. In Section 3, we will study the solution for (1.1) using the Mountain Pass Lemma. 


\section{Existence}

In this section, we state and prove the main theorem via Galerkin method when $\Omega$ is bounded. For convenience, we give the following hypotheses.

(H1) A typical assumption for $M$ is that there exists an $m_{0}>0$ such that $M(s) \geq m_{0}$, for all $s \geq 0$.

(H2) For all $s \in R$, assume that the functions $f, g$ satisfying

$$
\begin{array}{ll}
|f(x, s)| \leq C_{1}\left(1+|s|^{p_{1}-1}\right), & \text { a.e. in } \Omega, \\
|g(x, s)| \leq C_{2}\left(1+|s|^{p_{2}-1}\right), & \text { a.e. on } \partial \Omega,
\end{array}
$$

where $C_{1}, C_{2}>0$ are constants, $2<p_{1}<2^{*}=2 N /(N-2), 2<p_{1}<2^{*}=2(N-$ $1) /(N-2)$.

(H3) The function $x \mapsto f(x, 0)+g(x, 0)$ is not identically zero.

Let $W^{1,2}(\Omega)=\left\{u \in L^{2}(\Omega): \nabla u \in L^{2}(\Omega)\right\}$ be endowed with norm $\|u\|^{2}=\int_{\Omega}\left(|\nabla u|^{2}+\right.$ $\left.|u|^{2}\right) d x$. Then $W^{1,2}(\Omega)$ is a Banach space.

A function $u \in W^{1,2}(\Omega)$ is a weak solution of (1.1) if

$$
\int_{\Omega} \nabla u \nabla \varphi d x+\int_{\Omega} u \varphi d x-\int_{\partial \Omega} g(x, u) \varphi d x=\int_{\Omega} \frac{f}{\left.M(\| u) \|^{2}\right)} \varphi d x
$$

for all $\varphi \in W^{1,2}(\Omega)$.

Lemma 2.1. Suppose that $F: R^{m} \rightarrow R^{m}$ is a continuous function such that $\langle F(\xi), \xi\rangle \geq 0$ on $|\xi|=r$, where $\langle\cdot, \cdot\rangle$ is the usual inner product in $R^{m}$ and $|\cdot|$ its related norm. Then, there exists $z_{0} \in \overline{B_{r}}(0)$ such that $F\left(z_{0}\right)=0$.

Lemma 2.2 (see [16]). Let $\Omega$ be a domain in $R^{n}$ satisfying the uniform $C^{m}$-regularity condition, and suppose that there exists a simple $(m, p)$-extension operator $E$ for $\Omega$. Also suppose that $m p<n$ and $p \leq q \leq p^{*}=(n-1) p /(n-m p)$. Then

$$
W^{m, p}(\Omega) \hookrightarrow L^{q}(\partial \Omega)
$$

If $m p=n$, then the embedding still holds for $p \leq q<\infty$. Moreover, if $1<p \leq q<p^{*}$, then the embedding is compact.

Theorem 2.3. Assume that (H1)-(H3) hold. In addition, we suppose that

(H4) there exist constants $\lambda, \eta, \mu, C_{3}$ such that $f(x, u) u \leq \lambda|u|^{2}+\eta|u|, \forall x \in \Omega, u \in$ $R, g(x, u) u \leq \mu\left(C_{3} m_{0}\right)^{-1}|u|^{2}, \forall x$ on $\partial \Omega$ with

$$
\lambda+\mu<m_{0}
$$


Then problem (1.1) has at least one weak solution. Besides, any solution u satisfies the estimate

$$
\|u\| \leq \frac{m_{0} \eta|\Omega|^{1 / 2}}{\left(m_{0}-\lambda-\mu\right)}
$$

Proof. Let $\left\{\psi_{k}\right\}$ be different complete orthonormal systems for $W^{1,2}(\Omega)$ and set

$$
V_{n}=\operatorname{Span}\left\{\psi_{1}, \ldots, \psi_{n}\right\}
$$

Then $V_{n}$ is isometric to $R^{n}$. Then, each $u \in V_{n}$ is uniquely associated to $\xi=\left(\xi_{1}, \ldots, \xi_{n}\right)$ by the relation $u=\sum \xi_{k} \varphi_{k}$. Since $\left\{\psi_{k}\right\}$ are, respectively, orthonormal in $W^{1,2}(\Omega)$, we get $\|u\|^{2}=\|\xi\|_{R^{n}}^{2}$. We search for solutions $u_{n} \in V_{n}$ of the approximate problem

$$
\begin{array}{r}
\int_{\Omega} \nabla u_{n} \nabla \psi_{k} d x+\int_{\Omega} u_{n} \psi_{k} d x-\int_{\Omega} \frac{f\left(x, u_{n}\right)}{M\left(\left\|u_{n}\right\|^{2}\right)} \psi_{k} d x-\int_{\partial \Omega} g\left(x, u_{n}\right) \psi_{k} d y=0 \\
\forall \psi_{k} \in W^{1,2}(\Omega), \quad k=1,2, \ldots, n .
\end{array}
$$

To solve this algebraic system we define the operator $P_{n}: R^{n} \rightarrow R^{n}$

$$
\begin{aligned}
\left(P_{n} u\right)_{k}= & \int_{\Omega} \nabla u_{n} \nabla \psi_{k} d x+\int_{\Omega} u_{n} \psi_{k} d x-\int_{\Omega} \frac{f\left(x, u_{n}\right)}{M\left(\left\|u_{n}\right\|^{2}\right)} \psi_{k} d x \\
& -\int_{\partial \Omega} g\left(x, u_{n}\right) \psi_{k} d y=0, \quad \forall u \in V_{n} .
\end{aligned}
$$

By condition (H2), the growth of function $f$ is subcritical, so $u \mapsto f(\cdot, u)$ defines a continuous Nemytskii mapping $N_{f}: L^{p_{1}}(\Omega) \rightarrow L^{p_{1}^{\prime}}(\Omega)$. Similarly, we also define a continuous mapping $N_{g}: L^{p_{2}}(\Omega) \rightarrow L^{p_{2}^{\prime}}(\Omega)$.

From the continuity of $M$ and $f(x, u), g(x, u)$, with respect to $u$, we denote that $P_{n}$ is continuous. Therefore, from (H1), (H2), (H4) and Hölder's inequality, we note that $u \in V_{n}$

$$
\left\langle P_{n} u, u\right\rangle \geq\|u\|^{2}-\frac{\lambda\|u\|^{2}+\eta|\Omega|^{1 / 2}\|u\|}{m_{0}}-\int_{\partial \Omega}\left(\frac{\mu}{m_{0} C_{3}}|u|^{2}\right) d y .
$$

On the other hand, by Lemma 2.2, we have

$$
\int_{\partial \Omega}\left(\mu C_{3}^{-1} m_{0}^{-1}|u|^{2}\right) d y=\mu C_{3}^{-1} m_{0}^{-1}\|u\|_{L^{2}(\partial \Omega)}^{2} \leq \mu\left(C_{3} m_{0}\right)^{-1} C_{3}\|u\|^{2}=\frac{\mu}{m_{0}}\|u\|^{2},
$$

where $C_{3}>0$ is constant.

From (2.9) and (2.10), we can prove that

$$
\left\langle P_{n} u, u\right\rangle \geq\left(1-\frac{\lambda+\mu}{m_{0}}\right)\|u\|^{2}-\frac{\eta}{m_{0}}|\Omega|^{1 / 2}\|u\| .
$$


This shows that there exists $R>0$, depending only on $m_{0}, \lambda, \eta, \mu, C_{3}, \Omega$, such that $\left\langle P_{n} u, u\right\rangle \geq 0$ if $\|u\|=R$. Then system (2.7) has a solution $u_{n} \in V_{n}$ satisfying

$$
\left\|u_{n}\right\| \leq R
$$

From this bound estimate, going to a subsequence if necessary, there are $v$ and $u$ such that

$$
\left\|u_{n}\right\|^{2} \longrightarrow v, \quad u_{n} \rightarrow u \text { weakly in } W^{1,2}(\Omega)
$$

Besides, since $W^{1,2}(\Omega) \hookrightarrow L^{p_{1}}(\Omega), W^{1,2}(\Omega) \hookrightarrow L^{p_{2}}(\partial \Omega)$ compactly and the mapping $N_{f}, N_{g}$ is, respectively, continuous $L^{p_{1}}(\Omega) \rightarrow L^{p_{1}^{\prime}}(\Omega)$ and $L^{p_{2}}(\partial \Omega) \rightarrow L^{p_{2}^{\prime}}(\partial \Omega)$

$$
\begin{array}{cl}
u_{n} \longrightarrow u \text { in } L^{p_{1}}(\Omega), & f\left(x, u_{n}\right) \longrightarrow f(x, u) \quad \text { in } L^{p_{1}^{\prime}}(\Omega), \\
u_{n} \longrightarrow u \text { in } L^{p_{2}}(\partial \Omega), & g\left(x, u_{n}\right) \longrightarrow g(x, u) \quad \text { in } L^{p_{2}^{\prime}}(\partial \Omega) .
\end{array}
$$

Then fixing $k$ in (2.7) and letting $n \rightarrow \infty$, we conclude that

$$
\int_{\Omega} \nabla u \nabla \psi_{k} d x+\int_{\Omega} u \psi_{k} d x-\int_{\Omega} \frac{f(x, u)}{M(v)} \psi_{k} d x-\int_{\partial \Omega} g(x, u) \psi_{k} d y=0
$$

From the completeness of $\psi_{k}$, identity holds with $\psi_{k}$ replaced by any $\psi \in W^{1,2}(\Omega)$. In particularly, when $\psi=u$, we get

$$
\int_{\Omega} \nabla u \nabla \psi_{k} d x+\int_{\Omega} u \psi_{k} d x-\int_{\Omega} \frac{f(x, u)}{M(v)} \psi_{k} d x-\int_{\partial \Omega} g(x, u) \psi_{k} d y=0
$$

On the other hand, let $\psi_{k}=u_{n}$ in (2.7) and passing to the limit, we get

$$
v-\int_{\Omega} \frac{f(x, u) u}{M(v)} d x-\int_{\partial \Omega} g(x, u) u d y=0
$$

Then we conclude that $v=\|u\|^{2}$, which shows that $u$ is a solution of (1.1). Finally, if $u$ is any solution of (1.1) and $u$ is nontrivial, then

$$
\begin{gathered}
\|u\|^{2}-\int_{\Omega} \frac{f(x, u) u}{M\left(\|u\|^{2}\right)} d x-\int_{\partial \Omega} g(x, u) u d y=0, \\
\|u\| \leq \frac{\eta|\Omega|^{1 / 2}}{\left(m_{0}-\lambda-\mu\right)} .
\end{gathered}
$$

The proof is complete. 


\section{Variational Method}

In this section, we consider the following problem:

$$
\begin{gathered}
M\left(\int_{\Omega}|\nabla u|^{2}+|u|^{2} d x\right)(-\Delta u+u)=a u^{p_{1}-1}+b, \quad \text { in } \Omega, \\
\frac{\partial u}{\partial \gamma}=c u^{p_{2}-1}+d, \quad \text { on } \partial \Omega
\end{gathered}
$$

where $a, b, c, d$ are constants, and $p_{1}, p_{2}$ are defined in (H2).

The nontrivial solution of (3.1) comes from the Mountain Pass Lemma in [17].

Lemma 3.1 (Mountain Pass Lemma). Let $E$ be a Banach space and let $I \in C^{1}(E, R)$ satisfy the Palais-Smale condition. Suppose also that

(i) $I(0)=0$,

(ii) there exist constants $r, a>0$ such that $I(u) \geq a$, if $\|u\|=r$,

(iii) there exists an element $v \in H$ with $\|v\|>r, I(v) \leq 0$.

Define $\Gamma:=\{g \in C[0,1] ; H: g(0)=0, g(1)=v\}$. Then

$$
c=\inf _{g \in \Gamma} \max _{0 \leq t \leq 1} I[g(t)]
$$

is a critical value of $I$.

Theorem 3.2. Assume the conditions (H1)-(H3) hold. In addition, the function M satisfies

(H5) there exist $m_{1} \geq m_{0}$ with $\left(m_{0} / 2\right)-\left(m_{1} / p\right)>0$ and $t_{0}>0$, such that $M(t)=m_{1}, \forall t \geq t_{0}$, where $p=\min \left\{p_{1}, p_{2}\right\}$.

Then (3.1) has a nontrivial solution.

Proof. The weak solutions of (3.1) are critical points of the functional $J: W^{1,2}(\Omega) \rightarrow R$ defined by

$$
J(u)=\frac{1}{2} \widehat{M}\left(\|u\|^{2}\right)-\frac{1}{p_{1}} \int_{\Omega} a u^{p_{1}} d x-\frac{1}{p_{2}} \int_{\partial \Omega} c u^{p_{2}} d y-\int_{\Omega} b u d x-\int_{\partial \Omega} d u d y,
$$

where $\widehat{M}(t)=\int_{0}^{t} M(s) d s$.

Let us check the $(P S)$ condition. Let $\psi \in W^{1,2}$, we have

$$
\begin{aligned}
J^{\prime}(u) \psi= & M\left(\|u\|^{2}\right)\left(\int_{\Omega}(\nabla u \nabla \psi+u \psi) d x\right)-\int_{\Omega} a u^{p_{1}-1} \psi d x \\
& -\int_{\partial \Omega} c u^{p_{2}-1} \psi d y-\int_{\Omega} b \psi d x-\int_{\partial \Omega} d \psi d y .
\end{aligned}
$$


Let $\left\{u_{n}\right\}$ be a Palais-Smale sequence in $W^{1,2}(\Omega)$, that is, $J\left(u_{n}\right) \rightarrow \bar{c}$ and $J^{\prime}\left(u_{n}\right) \rightarrow 0$ and assume the contradiction that $\left\|u_{n}\right\| \rightarrow+\infty$, then, from (H1), (H5), we have

$$
\begin{aligned}
J\left(u_{n}\right)-\frac{1}{p} J^{\prime}\left(u_{n}\right) u_{n} \geq & \left(\frac{m_{0}}{2}-\frac{m_{1}}{p}\right)\left\|u_{n}\right\|^{2}-\left(\frac{1}{p_{1}}-\frac{1}{p}\right) \int_{\Omega} a u_{n}^{p_{1}} d x \\
& -\left(\frac{1}{p_{2}}-\frac{1}{p}\right) \int_{\partial \Omega} c u_{n}^{p_{2}} d y-\left(1-\frac{1}{p}\right) \int_{\Omega} b u_{n} d x-\left(1-\frac{1}{p}\right) \int_{\partial \Omega} d u_{n} d y,
\end{aligned}
$$

where $a, b, c, d>0$. Then by the Sobolev embedding theorem and Lemma 2.2, we can select $C>0$ such that

$$
C+C\left\|u_{n}\right\| \geq m_{0}\left(\frac{1}{2}-\frac{1}{p}\right)\left\|u_{n}\right\|^{2}
$$

which is a contradiction with $\left\|u_{n}\right\| \rightarrow+\infty$. Hence $\left\{u_{n}\right\}$ is bounded in $W^{1,2}(\Omega)$. So $\left\{u_{n}\right\}$ admits a weakly convergence subsequence. From (H2), all the growth of $f$, gis subcritical, so the standard argument shows that $\left\{u_{n}\right\}$ admits a strongly convergence subsequence.

Next we will verify the hypotheses of Lemma 3.1. By Hölder's inequality, Sobolev embedding theorem, and Lemma 2.2, we have

$$
\begin{gathered}
\int_{\Omega} a|u|^{p_{1}} d x=a\|u\|_{L^{p_{1}(\Omega)}}^{p_{1}} \leq N_{1}\|u\|^{p_{1}}, \quad \int_{\partial \Omega} c|u|^{p_{2}} d y=c\|u\|_{L^{p_{2}(\partial \Omega)}}^{p_{2}} \leq N_{2}\|u\|^{p_{2}}, \\
\int_{\Omega} b|u| d x \leq b N_{3}\|u\|, \quad \int_{\partial \Omega} d|u| d y \leq d N_{4}\|u\| .
\end{gathered}
$$

So we obtain

$$
J(u) \geq \frac{1}{2} m_{0}\|u\|^{2}-N_{1}\|u\|^{p_{1}}-N_{2}\|u\|^{p_{2}}-b N_{3}\|u\|-d N_{4}\|u\| .
$$

Let $\|u\|<1$, we get

$$
J(u) \geq \frac{1}{2} m_{0}\|u\|^{2}-N_{5}\|u\|^{p}-b N_{3}\|u\|-d N_{4}\|u\| .
$$

Let $h(r)=(1 / 2) m_{0} r^{2}-N_{5} r^{p}-N_{6} \varepsilon \cdot r$, then we take $r=r_{0}=3 N_{6} \varepsilon / 2 m_{0}$ such that $h\left(r_{0}\right)=\bar{a}=$ $\left(3 N_{6}^{2} / m_{0}\right) \varepsilon^{2}-N_{5} 3^{P} N_{6}^{p} \varepsilon^{p}>0$, when $\varepsilon$ is sufficient small.

So for $b$ and $d$ small enough, then we have $J(u) \geq \bar{a}>0$ for all $\|u\|=r_{0}$. 
On the other hand, take $\omega_{0} \in W^{1,2}(\Omega)$ with $\int_{\Omega} a \omega_{0}^{p_{1}} d x=1$ for $k>0$, we have

$$
\begin{aligned}
J\left(k \omega_{0}\right) & =\frac{1}{2} \widehat{M}\left(\left\|k \omega_{0}\right\|^{2}\right)-\frac{k^{p_{1}}}{p_{1}} \int_{\Omega} a \omega_{0}^{p_{1}} d x-\frac{1}{p_{2}} \int_{\partial \Omega} c\left(k \omega_{0}\right)^{p_{2}} d y-\int_{\Omega} b k \omega_{0} d x-\int_{\partial \Omega} k d \omega_{0} d y \\
& \leq \frac{1}{2} m_{1}\left\|k \omega_{0}\right\|^{2}-\frac{k^{p_{1}}}{p_{1}} \int_{\Omega} a \omega_{0}^{p_{1}} d x-\frac{1}{p_{2}} \int_{\partial \Omega} c\left(k \omega_{0}\right)^{p_{2}} d y-\int_{\Omega} b k \omega_{0} d x-\int_{\partial \Omega} k d \omega_{0} d y
\end{aligned}
$$

Since $p_{1}, p_{2}>2$, we obtain $J\left(k \omega_{0}\right) \rightarrow-\infty$ when $k \rightarrow+\infty$.

Let $\omega=k \omega_{0}$, with $k$ large enough, we have $\|\omega\|>\max \left\{t_{0}, r_{0}\right\}$ and $J(\omega)<\bar{a}$. So by the Mountain Pass Lemma and (H3), we have a nontrivial solution $u(x)$ for (3.1). The proof is complete.

\section{References}

[1] G. Kirchhoff, Mechanik, Teubner, Leipzig, Germany, 1883.

[2] J.-L. Lions, "On some questions in boundary value problems of mathematical physics," in Contemporary Developments in Continuum Mechanics and Partial Differential Equations (Proc. Internat. Sympos., Inst. Mat., Univ. Fed. Rio de Janeiro, Rio de Janeiro, 1977), G. de la Penha and L. A. Medeiros, Eds., vol. 30 of North-Holland Mathematics Studies, pp. 284-346, North-Holland, Amsterdam, The Netherlands, 1978.

[3] A. Arosio and S. Panizzi, "On the well-posedness of the Kirchhoff string," Transactions of the American Mathematical Society, vol. 348, no. 1, pp. 305-330, 1996.

[4] K. Ono, "On global solutions and blow-up solutions of nonlinear Kirchhoff strings with nonlinear dissipation," Journal of Mathematical Analysis and Applications, vol. 216, no. 1, pp. 321-342, 1997.

[5] C. O. Aives, F. J. S. A. Corrêa, and T. F. Ma, "Positive solutions for a quasilinear elliptic equation of Kirchhoff type," Computers \& Mathematics with Applications, vol. 49, no. 1, pp. 85-93, 2005.

[6] T. F. Ma, "Remarks on an elliptic equation of Kirchhoff type," Nonlinear Analysis: Theory, Methods $\mathcal{E}$ Applications, vol. 63, no. 5-7, pp. e1967-e1977, 2005.

[7] K. Perera and Z. Zhang, "Nontrivial solutions of Kirchhoff-type problems via the Yang index," Journal of Differential Equations, vol. 221, no. 1, pp. 246-255, 2006.

[8] Z. Zhang and K. Perera, "Sign changing solutions of Kirchhoff type problems via invariant sets of descent flow," Journal of Mathematical Analysis and Applications, vol. 317, no. 2, pp. 456-463, 2006.

[9] R. Stańczy, "Nonlocal elliptic equations," Nonlinear Analysis: Theory, Methods E Applications, vol. 47, no. 5, pp. 3579-3584, 2001.

[10] F. J. S. A. Corrêa and D. C. de Morais Filho, “On a class of nonlocal elliptic problems via Galerkin method," Journal of Mathematical Analysis and Applications, vol. 310, no. 1, pp. 177-187, 2005.

[11] J. F. Bonder and J. D. Rossi, "Existence results for the $p$-Laplacian with nonlinear boundary conditions," Journal of Mathematical Analysis and Applications, vol. 263, no. 1, pp. 195-223, 2001.

[12] K. Chaïb, "Necessary and sufficient conditions of existence for a system involving the $p$-Laplacian $(1<p<N)$," Journal of Differential Equations, vol. 189, no. 2, pp. 513-525, 2003.

[13] P. Amster, M. C. Mariani, and O. Méndez, "Nonlinear boundary conditions for elliptic equations," Electronic Journal of Differential Equations, vol. 2005, no. 144, pp. 1-8, 2005.

[14] S.-Z. Song and C.-L. Tang, "Resonance problems for the $p$-Laplacian with a nonlinear boundary condition," Nonlinear Analysis: Theory, Methods E Applications, vol. 64, no. 9, pp. 2007-2021, 2006.

[15] J.-H. Zhao and P.-H. Zhao, "Existence of infinitely many weak solutions for the $p$-Laplacian with nonlinear boundary conditions," Nonlinear Analysis: Theory, Methods E Applications, vol. 69, no. 4, pp. 1343-1355, 2008.

[16] R. A. Adams and J. J. F. Fournier, Sobolev Spaces, Academic Press, Amsterdam, The Netherlands, 2003.

[17] L. C. Evans, Partial Differential Equations, American Mathematical Society, Providence, RI, USA, 1998. 